\title{
Possible relationship between certolizumab pegol and arrhythmias: report of two cases
}

\author{
R. Talotta, F. Atzeni, A. Batticciotto, D. Ventura, P. Sarzi-Puttini \\ Rheumatology Unit, Luigi Sacco University Hospital, Milan, Italy
}

\section{SUMMARY}

It is still unknown whether there is an association between the use of certolizumab pegol (CZP) in rheumatic patients and the onset of cardiac arrhythmias. We describe the cases of two patients with rheumatoid arthritis (RA) treated with CZP as the first-line biological drug and methotrexate (MTX), who developed an arrhythmic event. The first was a 60-year-old, hypertensive male smoker, the second a 66-year-old dyslipidemic female non-smoker. Both were diagnosed as having RA in 2010, and started treatment with MTX plus CZP. The first patient developed undatable atrial fibrillation, which was resistant to pharmacological treatment and electrical cardioversion. The second patient developed an atrial flutter, which was treated with a betablocker. In both cases, we set a cautious interval between two consecutive administrations of CZP and, in the first case, also reduced the dose of MTX without any worsening of RA activity. Although many studies have shown that tumor necrosis factor (TNF)-alpha plays a pathogenetic role in inducing an arrhythmogenic substrate that is apparently rescued by anti-TNF drugs, there is still a lack of conclusive data. We suggest caution in any patient developing a cardiac event (including rhythm disorders) during treatment with a conventional or biological disease-modifying anti-rheumatic drug.

Key words: Anti-TNF drugs; heart diseases; rheumatoid arthritis; arrhythmia.

Reumatismo, 2016; 68 (2): 104-108

\section{INTRODUCTION}

The anti-tumour necrosis factor-alpha (anti-TNF $\alpha$ ) agents approved for the treatment of rheumatoid arthritis (RA) and spondyloarthritis (SpA) are biological drugs with well-characterized safety and efficacy profiles. Certolizumab pegol $(\mathrm{CZP})$ is a pegylated antigen-binding fragment of a humanized monoclonal antibody against TNF $\alpha$ that is licensed for use in patients with RA or $\operatorname{SpA}(1,2)$. Because of its molecular structure, CZP rapidly reaches inflamed joints and provides symptomatic relief from the time of its first administrations (3).

TNF $\alpha$ plays a crucial role in remodelling acutely or chronically damaged heart tissue. However, administering an anti-TNF $\alpha$ agent in patients with cardiac disorders may be simultaneously useful and detrimental as cases of precipitating heart failure and arrhythmias have been reported during treatment with infliximab, etaner- cept and adalimumab, whose use is therefore contraindicated in patients with New York Heart Association class III and IV heart failure. No data have yet been published about the risk of CZP-induced arrhythmia, which is described as being an uncommon side effect in drug data sheets and pharmacovigilance reports.

We describe two cases of cardiac arrhythmias occurring during treatment with CZP as the first biological line in two RA patients.

\section{CASE REPORTS}

\section{Case \#1}

The first patient was a 60-year-old, hypertensive male smoker whose hypertension had been treated with an angiotensin-converting enzyme inhibitor, a thiazide diuretic (enalapril/hydrochlorothiazide 20/12.5 $\mathrm{mg}$ /day), a calcium channel antagonist (lacidipine $4 \mathrm{mg} / \mathrm{day}$ ) and a beta-blocker (bisoprolol $1.25 \mathrm{mg} /$ day) from the age of 
42 years. He began experiencing peripheral arthralgias in October 2010 and was referred to our center. At the first visit the patient had both wrists and knees tender and swollen and laboratory tests revealed the presence of anti-citrullinated peptide antibodies and rheumatoid factor. A diagnosis of early RA (4) was made and treatment with methotrexate (MTX) $15 \mathrm{mg} /$ week associated with prednisone $5 \mathrm{mg} /$ daily was promptly prescribed, which led to remission lasting for nearly two years. However, in July 2012, the patient experienced an arthritic flare [8 swollen joints, 9 tender joints, erythrocyte sedimentation rate $(\mathrm{ESR})=22 \mathrm{~mm}^{\text {st }} \mathrm{h}, \mathrm{C}$-reactive protein $(\mathrm{CRP})=3.4 \mathrm{mg} / \mathrm{L}$, disease activity score on 28 joints $($ DAS28) $=4.971$ and due to the partial efficacy of MTX, after a careful screening for the use of anti-TNF drugs, CZP $200 \mathrm{mg}$ every other week was added in November 2012.

The combined treatment led to a rapid improvement in clinical and laboratory parameters, although it had less effect on auto-antibody titres.

The baseline clinical examination revealed a bradycardic rhythm probably related to the use of the beta-blocker and, in October 2013, about one year after starting CZP, he was admitted to the Emergency Department because of an undatable episode of atrial fibrillation (AF) that failed to respond to anti-arrhythmic treatment with amiodarone and flecainide. Echocardiography revealed left atrial dilatation (48 $\mathrm{mm}$ ) and moderate mitral and tricuspid valve insufficiency.

The patient immediately started treatment with an oral anti-coagulant (warfarin) and, after three attempts at electrical cardioversion in January, February and March 2014, sinus rhythm was eventually restored. Treatment with amiodarone $100 \mathrm{mg} /$ day was continued until April 2015, when it was stopped by the patient, and AF re-occurred in May 2015.

Meanwhile, given the clinical remission of RA and the potential risk of using an anti-TNF agent during a cardiac event, the interval between CZP injections was extended to three weeks in May 2014 (when the dose of MTX was lowered to $7.5 \mathrm{mg} /$ week) and then to four weeks in October of the same year.

The patient is currently still arrhythmic (slow heart rate $\mathrm{AF}$ ) and receiving a treatment with bisoprolol $1.25 \mathrm{mg} /$ day and apixaban $10 \mathrm{mg} /$ day. His anti-hypertensive treatment has been maintained, and the continued treatment with MTX $7.5 \mathrm{mg} /$ week and CZP $200 \mathrm{mg}$ every 28 days is still ensuring good control of the rheumatic disease (DAS28=1.79 in June 2015).

\section{Case \#2}

The second patient was a 66-year-old nonsmoker female, who had diet-treated dyslipidemia since 2001. In March 2010, she was diagnosed as having seropositive RA and treatment with MTX $15 \mathrm{mg} /$ week was started. Concomitantly, she reported periodic extra-systoles and anxiety symptoms that were treated with bromazepam as needed. Echocardiography performed in 2010 for a suspected organic heart disease only revealed mitral valve prolapse.

As RA disease activity was only partially controlled ( 7 swollen and tender joints, $\mathrm{ESR}=58 \mathrm{~mm}^{1 \mathrm{st}} \mathrm{h}, \mathrm{CRP}=5.3 \mathrm{mg} / \mathrm{L}$, DAS28=5.62), CZP $200 \mathrm{mg}$ every other week was added in June 2012. This was fairly successful clinimetrically, although there was little change in her auto-antibody titres. In December 2013, an increase in liver enzyme levels led to a reduction in the dose of MTX (7.5 mg every 10 days) and, in May 2015, hydroxychloroquine $200 \mathrm{mg} /$ day was added.

In June 2015, she attended the Emergency Department because of the onset of tachyarrhythmia, which proved to be an atrial flutter. A new echocardiography examination revealed initial left atrial dilatation, and treatment with an oral anticoagulant plus heparin and a beta-blocker (carvedilol $3.125 \mathrm{mg} /$ day) was prescribed. At the time of her last visit to our centre (June 2015), RA was in remission (DAS28=2.50); the treatment with conventional disease modifying antirheumatic drugs (DMARDs) was maintained, whereas the administration of CZP was tapered to one injection every 21 days. The 
patient is currently waiting to undergo radio-frequency ablation of the arrhythmogenic focus.

\section{DISCUSSION}

$\mathrm{TNF} \alpha$ plays a crucial role in cardiac tissue remodeling. The myocardiocytes of patients affected by heart failure express TNF $\alpha$ receptors and may also synthesize $\mathrm{TNF} \alpha$ which induces the activation of nuclear factor kappa-light-chain-enhancer of activated B cells and favors myocardiocyte proliferation and apoptosis, the deposition of extracellular matrix, and the up- or down-regulation of metalloproteases (MMP). It also increases the amount of intracytosolic calcium ions and the production of nitric oxide (5). The systemic inflammation occurring during the course of rheumatic diseases can also affect cardiac tissue and generate histological changes that increase the risk of arrhythmias. There is much evidence to support the pathogenetic role of cytokines such as TNF $\alpha$ and interleukin (IL)-6 in AF (6-8), and systemic inflammation, increased CRP levels and a pro-coagulant state are also risk factors for AF-related thromboembolism (9). In animal models, by binding to TNFRI and TNFRII on atrial myocardiocytes and fibroblasts, TNF $\alpha$ may increase the deposition of extracellular matrix, reduce the expression of connexin-40, and increase the release of MMP, thus finally contributing to atrial remodeling (10).

The use of anti-TNF $\alpha$ agents can be useful in reducing the inflammatory burden and restoring endothelial function, but may simultaneously interfere with left ventricular remodeling or induce the complementdependent cytotoxicity or antibody-dependent cell-mediated cytotoxicity of myocardiocytes. The neutralization of $\mathrm{TNF} \alpha$ induced by administering the monoclonal antibody infliximab to Trypanosoma cruziinfected mice restores cardiac inflammation by increasing the level of IL-10 while the decrease in IL-17 concentrations reduces the deposition of fibronectin, and thus prevents the risk of arrhythmias (11).

$\mathrm{TNF} \alpha$ also seems to affect the ion-potential currents of myocardiocytes. Experiments on mice treated with $\mathrm{TNF} \alpha$ have highlighted a reduced intake of sarcoplasmic $\mathrm{Ca} 2+$, possibly related to the nitrosylation of RyR2 channels $(12,13)$. This reduction in $\mathrm{Ca} 2+$ levels may be responsible for depressed cell contractility and the development of aberrant sparks or hump-like depolarizations, which are risk factors for the occurrence of an arrhythmic event. Patients with ankylosing spondylitis or inflammatory bowel diseases often have a prolonged QT interval (14), which, according to some authors, can be restored by treatment with infliximab (15). Neither of our patients had alterations of the QT interval, as emerged by an electrocardiographic test performed at baseline and after 12 month of treatment wit CZP. Murine models have shown that TNF $\alpha$ may promote exercise-induced atrial remodeling and favor the occurrence of $\mathrm{AF}$, both of which can be prevented by the use of etanercept (16). Moreover, the use of infliximab in RA patients seems to improve echocardiographic findings of diastolic dysfunction and left atrial volumes within the first three months of treatment (17).

A recent review of arrhythmic events in RA patients found an increased risk of sudden cardiac death (often related to malignant ventricular arrhythmias, a prolonged QT interval frequently associated with increased CRP levels, and AF) in comparison with the general population; some of these anomalies may be normalized by treatment with the biological agent tocilizumab (18). The higher incidence of arrhythmic events can be attributed to the detrimental effects of systemic inflammation on the myocardium and the triggering of the autonomous sympathetic system.

Few published reports have highlighted an association between the risk of developing cardiac arrhythmia and the concomitant treatment with a TNF $\alpha$ blocker (19-21), and this association seems to be stronger in RA patients with a prolonged QT interval (22). Accordingly, trials of anti-TNF agents during cardiac failure or post-infarction have not led to any conclusive findings and were stopped prematurely (23).

To the best of our knowledge, there are no 
published case reports of arrhythmia during treatment with CZP that, like other anti-TNF drugs, acts by blocking the remodeling effects of TNF $\alpha$. Although the apoptosis of myocardiocytes is less intense because of the absence of crystallizable fragments, it can be sustained by the activation of the pro-apoptotic pathway via TNFRI and TNFRII, or by means of the mechanism of reverse signaling, which has been demonstrated to be highly active during CZP therapy.

The safety of CZP has been tested in clinical trials and pre-pivotal placebo-controlled studies involving more than 4000 RA patients, and analyses of aggregate data and the information arising from postmarketing surveillance have classified the occurrence of arrhythmias (including AF) as uncommon (24). Furthermore, only three cases of tachycardia were reported in the CZP arm of the CERTAIN trial (25).

Both of our patients had a history of altered sinus rhythm (extrasystoles and bradycardia), and the concomitant use of MTX even at medium-low doses of 7.5-15 mg/week may have contributed to the development of arrhythmias, an association that is supported by some published alerting reports (26). Similarly, the concomitant use of hydroxychloroquine in patient \#2 may have been an aggravating factor, even though there are only rare reports of severe heart conduction disorders (bundle branch block/ atrioventricular block), during treatment with hydroxychloroquine, with complete remission after its discontinuation (27).

\section{CONCLUSIONS}

Our two RA patients experienced cardiac arrhythmias during combined CZP and MTX treatment. The interference of antiTNF agents with the physiological processes governing the correct function of the sinus node or heart conductance apparatus is still a matter of debate. However, given the number of reports of cardiac events developing during anti-TNF treatment, patients with RA and concomitant cardiac risk factors (e.g., extrasystoles, hypertension, or valvulopathies) should be referred to a cardiologist and assessed before and during treatment. In both cases, the development of the arrhythmic event may be explained by a predisposing organic condition and/or the use of conventional and biologic DMARDs that may have acted as a trigger. Once RA remission has been achieved, consideration should be given to tapering the dose or increasing the interval between two consecutive administrations of the TNF $\alpha$ blocker. Reducing the conventional DMARD burden may be an alternative choice.

Conflict of interest: the authors declare no potential conflict of interest.

Acknowledgements: we acknowledge all of the physicians in the Rheumatology Unit of Luigi Sacco University Hospital for giving us access to the patients' source documents.

\section{REFERENCES}

1. EH, Hazleman B, Smith M, et al. Efficacy of a novel PEGylated humanized anti-TNF fragment (CDP870) in patients with rheumatoid arthritis: a phase II double-blinded, randomized, dose-escalating trial. Rheumatology (Oxford). 2002; 41: 1133-7.

2. Dhillon S. Certolizumab pegol: a review of its use in patients with axial spondyloarthritis or psoriatic arthritis. Drugs. 2014; 74: 9991016.

3. Palframan R, Airey M, Moore A, et al. Use of biofluorescence imaging to compare the distribution of certolizumab pegol, adalimumab, and infliximab in the inflamed paws of mice with collagen-induced arthritis. J Immunol Methods. 2009; 348: 36-41.

4. Aletaha D, Neogi T, Silman AJ, et al. Rheumatoid arthritis classification criteria: an American College of Rheumatology/European League Against Rheumatism collaborative initiative. Arthritis Rheum. 2010; 62: 2569-81.

5. Sarzi-Puttini P, Atzeni F, Shoenfeld Y, Ferraccioli G. TNF-alpha, rheumatoid arthritis, and heart failure: a rheumatological dilemma. Autoimmun Rev. 2005; 4: 153-61.

6. Ren M, Li X, Zhong J. Role of tumor necrosis factor alpha in the pathogenesis of atrial fibrillation: a novel potential target? Ann Med. 2015; 47: 316-24.

7. Kimura T, Takatsuki S, Inagawa K, et al. Serum inflammation markers predicting successful initial catheter ablation for atrial fibrillation. Heart Lung Circ. 2014; 23: 636-43. 
8. Wu N, Xu B, Xiang Y, et al. Association of inflammatory factors with occurrence and recurrence of atrial fibrillation: a meta-analysis. Int J Cardiology. 2013; 169: 62-72.

9. Guo Y, Lip GYH, Apostolakis S. Inflammation in atrial fibrillation. J Am Coll Cardiol. 2012; 60: 2263-70.

10. R, Khairunnisa K, Gu Y, et al. Role of tumor necrosis factor-a and development of an arrhythmogenic substrate. Circulation J. 2013; 77: 1171-9.

11. Pereira IR, Vilar-Pereira G, Silva AA, et al. Tumor necrosis factor is a therapeutic target for immunological unbalance and cardiac abnormalities in chronic experimental Chagas' heart disease. Mediat Inflamm. 2014; 2014: 798078 .

12. Duncan DJ, Yang Z, Hopkins PM, et al. TNFleak from the sarcoplasmic reticulum and susceptibility to arrhythmia in rat ventricular myocytes. Cell Calcium. 2010; 47: 378-86.

13. Abramochkin DV, Kuzmin VS, Mitrochin VM, et al. TNF-a provokes electrical abnormalities in rat atrial myocardium via a NOdependent mechanism. Pflugers Arch-Eur J Physiol. 2013; 465: 1741-52.

14. Curione M, Aratari A, Amato S, et al. A study on QT interval in patients affected with inflammatory bowel disease without cardiac involvement. Intern Emerg Med. 2010; 5: 307-10.

15. Senel S, Cobankara V, Taskoylu O, et al. Effect of infliximab treatment on QT intervals in patients with ankylosing spondylitis. Investig Med. 2011; 59: 1273-5.

16. Aschar-Sobbi R, Izaddoustdar F, Korogyi AS, et al. Increased atrial arrhythmia susceptibility induced by intense endurance exercise in mice requires TNFs. Nat Commun. 2015; 6: 6018.

17. Suha C, Gokhan VM, Goksal K, et al. Infliximab, an anti-TNF-alpha agent, improves left atrial abnormalities in patients with rheumatoid arthritis: preliminary results. Cardiovascular J Africa. 2014; 25: 168-75.

18. Lazzerini PE, Capecchi PL, Acampa M, et al. Arrhythmic risk in rheumatoid arthritis: the driving role of systemic inflammation. Autoimmunity Rev. 2014; 13: 936-44.

19. Wooten MD, Reddy GV, Johnson RD. Atrial fibrillation occurring in a patient taking etanercept plus methotrexate for rheumatoid arthritis. Del Med J. 2000; 72: 517-9.

20. Eder P, Lykowska-Szuber L, Krela-Kazmierczak I, et al. Ventricular tachyarrhythmia after adalimumab therapy in a patient with Crohn's disease. Acta Gastroenterol Belg. 2013; 76: 74-5.

21. Sote Y, Green S, Maddison P. Complete heart block after infliximab therapy. Rheumatology (Oxford). 2008; 47: 227-8.

22. Lazzerini PE, Acampa M, Hammoud M, et al. Arrhythmic risk during acute infusion of infliximab: a prospective, single-blind, placebo-controlled, crossover study in patients with chronic arthritis. J Rheumatol. 2008; 35 : 1958-65.

23. Louis A, Cleland JG, Crabbe S, et al. Clinical Trials Update: CAPRICORN, COPERNICUS, MIRACLE, STAF, RITZ-2, RECOVER and RENAISSANCE and cachexia and cholesterol in heart failure. Highlights of the Scientific Sessions of the American College of Cardiology, 2001. Eur J Heart Fail. 2001; 3: 381-7.

24. Winter TA, Sandborn WJ, de Villiers WJ, Schreiber S. Treatment of Crohn's disease with certolizumab pegol. Expert Rev Clin Immunol. 2007; 3: 683-94.

25. Smolen JS, Emery P, Ferraccioli GF, et al. Certolizumab pegol in rheumatoid arthritis patients with low to moderate activity: the CERTAIN double-blind, randomised, placebo-controlled trial. Ann Rheum Dis. 2015; 74 : 843-50.

26. Perez-Verdia A, Angulo F, Hardwicke FL, Nugent KM. Acute cardiac toxicity associated with high-dose intravenous methotrexate therapy: case report and review of the literature. Pharmacotherapy. 2005; 25: 1271-6.

27. Tönnesmann E, Kandolf R, Lewalter T. Chloroquine cardiomyopathy - a review of the literature. Immunopharmacol Immunotoxicol. 2013; 35: 434-42. 